\title{
Norois
}

Environnement, aménagement, société

\section{Introduction - Vins, vignobles et viticultures atlantiques. Quelles trajectoires contemporaines?}

Introduction - Wines, vineyards and atlantic viticulture. Towards contemporary trajectories?

\section{Éric Rouvellac}

\section{(2) OpenEdition}

Journals

\section{Édition électronique}

URL : https://journals.openedition.org/norois/9577

DOI : $10.4000 /$ norois.9577

ISSN : $1760-8546$

\section{Éditeur}

Presses universitaires de Rennes

Édition imprimée

Date de publication : 30 juin 2020

Pagination : 7-19

ISBN : 978-2-7535-8119-7

ISSN : 0029-182X

\section{Référence électronique}

Éric Rouvellac, «Introduction - Vins, vignobles et viticultures atlantiques. Quelles trajectoires 


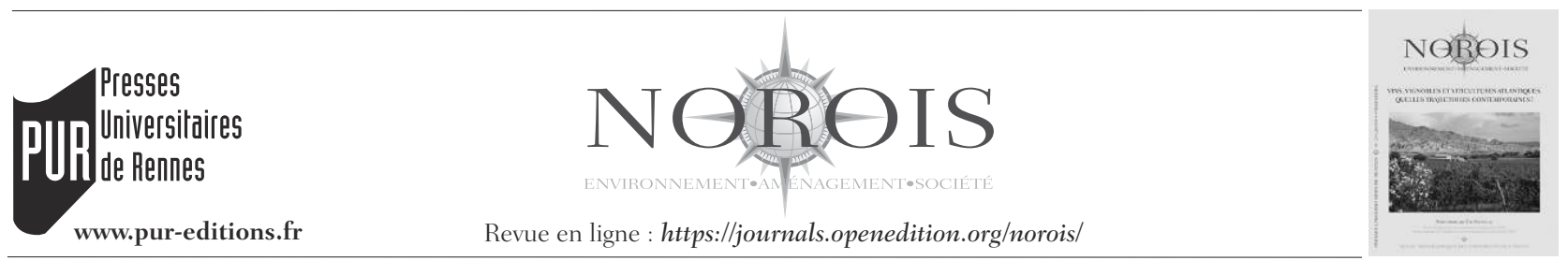

\title{
Introduction - Vins, vignobles et viticultures atlantiques. Quelles trajectoires contemporaines?
}

\author{
Introduction - Wines, vineyards and atlantic viticulture. \\ Towards contemporary trajectories?
}

\author{
Éric Rouvellac
}

Professeur de géographie, Université de Limoges, UMR 6042 GEOLAB (eric.rouvellac@unilim.fr)

L'histoire et la géographie de la vigne et du vin; le poids du vin dans les représentations, dans le patrimoine et dans l'identité de différents territoires, à différentes échelles géographiques; le prisme du réchauffement climatique, qui prend un relief particulier dans le monde vitivinicole : autant de sujets, non exhaustifs, auxquels est confronté le monde du vin d'aujourd'hui.

Traversé par des enjeux socio-économiques forts, où la mondialisation des marchés, des usages et des évolutions de consommation sont bousculés, l'Europe des vins de terroirs est confrontée depuis les années 1980 au Nouveau monde des vins de cépage. Mais n'observe-t-on un effet boomerang en retour? Ainsi, la culture des vins de terroirs revient-elle en Europe par les pays viticoles du Nouveau monde qui l'adoptent en même temps que nous développons les vins de marque et de cépage. De plus, certaines initiatives et certains territoires (y compris insulaires) essayent de tirer parti de l'effet de niche, une tendance actuelle garante pour le public d'une certaine qualité, à l'opposé de la standardisation, des circuits longs, du non-respect de l'environnement.
Depuis 1950, la majeure partie des vignobles du monde est confrontée à la problématique du réchauffement climatique (Jones et al., 2005, Quenol, 2014). Si les terroirs traditionnels doivent faire face à la question de l'adaptation, le réchauffement propose des opportunités à des espaces géographiques jusqu'ici réputés impropres à cette culture. Le modèle English and Welsh Viticulture Suitability estime par exemple le potentiel britannique en termes de surface favorable à la viniculture à 33700 ha (Nesbitt et al., 2018), soit presque autant que le vignoble champenois. Plus au sud, en Bretagne, à l'initiative de passionnés, on assiste ici et là au retour de vignobles, disparus progressivement à partir du XVI ${ }^{\mathrm{e}}$ siècle : en Ille-et-Vilaine, dans le Finistère (coteaux du Braden à Quimper), les côtes d'Armor ou dans le Morbihan, y compris dans les îles du Ponant. Que ce soit sous l'effet du changement climatique ou sous l'impulsion des communautés locales, le fait viticole s'inscrit dans des logiques d'évolution de son emprise.

La revue Norois ouvre ses pages à deux numéros thématiques sur la question viti-vinicole atlantique, 
à différentes échelles, depuis les côtes américaines jusqu'aux rives européennes et africaines. Il s'agit tout autant de dresser le portrait de vignobles littoraux existants ou disparus que d'examiner les trajectoires possibles, passées ou à venir de cette activité dans l'espace concerné. Si les questions du changement climatique et l'adaptation à ces changements (Lereboullet A.-L. 2014) sont importantes, elles convoquent d'autres approches du fait viticole : sur les terroirs, les marchés, la production, les transports, la consommation, le développement local, etc., sans oublier les questions de perceptions, de représentations, ou d'imaginaire.

L'appel à articles était donc volontairement large afin de mobiliser des auteurs et des contributions représentatives de la recherche en géographie et plus largement en sciences humaines et sociales sur le vin et les vignobles atlantiques. Et si les thèmes relatifs à la fabrique des paysages viticoles, liés ou non au concept de terroir, et de l'œnotourisme n'ont pas trouvé d'écho ici, les autres seront développés au gré des deux numéros, notamment à travers l'histoire des vignobles, la commercialisation des vins et les implications du réchauffement climatique dans celui-ci.

\section{Vins eT VIgNObLES ATLANTIQUES ENTRE HÉRITAGES ET RENOUVEAUX}

\section{Des origines du vin et des vignobles, le poids des représentations}

Pour des raisons liées intrinsèquement à son essence et à son histoire, le vin, comme d'autres produits, est un psychotrope qui permet à l'être humain de se projeter un cours moment, avec risques et périls parfois, en dehors de sa condition, par l'intermédiaire fugace de l'ivresse.

C'est une particularité qui le projette dans une vision transcendantale pour une succession de civilisations depuis le Néolithique, particularité dont il nous reste des traces par les cultes dionysiaques et bachiques. Pétrone dans son « Satiricon », n'écrivaitil pas que «la vie, c'est du vin »(vita vinum est), pour ponctuer les grandes fêtes qu'il donnait dans la Rome du $1^{\text {er }}$ siècle de l'empereur Néron?

Particularité qui s'exprime depuis deux millénaires par les cultes chrétiens où le mythe et l'histoire se répondent. Dans l'Ancien Testament, Noé arrive après le périple du déluge au sommet du mont
Ararat, plante de la vigne, et s'enivre. Le mont Ararat, dans l'actuelle Turquie, est compris dans ce grand berceau initial du Proche-Orient et des pourtours de la Mer Noire. Le vin et l'ivresse encore païenne de Noé trouvent un nouveau débouché retentissant dans la Cène, où le vin devient le sang du Christ par transsubstantiation. Il ne symbolise pas, il est le sang du prophète exprimant la résurrection dans le culte chrétien. Ce qui explique que chaque jour, l'officiant a besoin de vin pour la messe, et dans un monde où jusqu'à la révolution ferroviaire, transporter des pondéreux comme des liquides est très compliqué, le choix de planter les vignes à proximité est le seul possible pour rendre le culte.

Le vin garde son atout de chemin vers l'ivresse, auréolé de courants religieux qui vont diffuser la vigne de la Méditerranée aux rivages atlantiques de l'Europe, puis des Amériques et de l'Afrique australe.

De ce contexte de boisson psychotrope et religieuse découle une symbolique très forte qui confère à ce produit une valeur économique à la hauteur de l'enjeu. Le vin devient un produit commercial à forte valeur ajoutée, y compris dans des sociétés où l'économie vivrière est encore quasi généralisée. Derrière le monde du vin se trouve tout un secteur économique, parmi les seuls excédentaires à l'export pour la France, toute une filière de production, d'acteurs, de produits allant de la production de masse à l'exceptionnel, affichant des prix bas à très élevés.

La vigne est une liane tempérée qui trouve son terrain d'origine, sous forme cultivée, dans des climats plutôt méditerranéens, mais qui pousse facilement, d'autant plus avec l'aide du viticulteur, pratiquement sous tous les climats. Seules les régions les plus froides du tempéré nord, où les parties intertropicales les plus arrosées lui sont inhospitalières. Et encore trouve-t-on des initiatives qui parient sur l'effet de niche et sur le capital de l'étrangeté avec de la viticulture en Polynésie française sur lîle de Rangiroa, ou en Norvège dans la commune de Søgne dans l'extrême sud du pays, (le Figaro - vin, 29 mars 2020). Les Français ont essayé de l'introduire en Nouvelle-Calédonie dans les années 1930, les Anglais au Kenya, également lors de la colonisation; la vigne pousse et fructifie, mais la qualité n'est pas au rendez-vous.

Plus traditionnellement, la vigne s'est diffusée dans des terrains potentiellement plus productifs au gré des pérégrinations des proches Orientaux et des 
Européens le long des 3 derniers millénaires, avec une grande accélération lors des grandes découvertes de l'ère moderne, notamment dans la zone atlantique qui nous concerne.

Il n'est pas étonnant dans ces conditions de trouver le vin comme un élément de communication important dans la sensibilisation au changement climatique, pour frapper les esprits, alors que ce n'est pas un produit nécessaire à la survie alimentaire d'Homo sapiens. Mais peut-être est-il nécessaire à une vie qui n'est pas que matérielle, qui demeure aussi sociale et/ou spirituelle, avec un important bagage patrimonial, civilisationnel. Donc, quand les vignobles sont menacés de changements de lieu, voire de disparition, le public est supposé prendre davantage conscience des enjeux que pour des productions plus communes, vu le poids des représentations associées à la vigne et au vin.

Nous nous pencherons en premier lieu sur un exemple de territoire presque banni de la mémoire vitivinicole et qui, au nom de la diffusion eucharistique du vin, l'a été de manière importante du Moyen Âge jusqu'aux Temps modernes, la Bretagne. La vigne s'y est diffusé comme dans tous les territoires les plus océaniques du nord-ouest de l'Europe, où le climat n'est pas le plus propice, et s'est même fixé logiquement dans la périphérie des villes qui constituaient le premier marché local. Bien des cités, comme Paris par exemple, ont vu leur vignoble périurbain disparaître avec les échanges croissants aux XVIII ${ }^{\mathrm{e}}$ et XIX ${ }^{\mathrm{e}}$ siècles. Aujourd'hui dans un département quasi a-viticole comme la Haute-Vienne, une ville moyenne comme Limoges entretenait une superficie en vigne importante autour de ces murs figurant toujours sur son plan du XVII ${ }^{\mathrm{e}}$ siècle. C'est donc potentiellement tout le nord-ouest de l'Europe qui est concerné aujourd'hui par la (re)découverte de son histoire vitivinicole.

Dans ces régions atlantiques les plus arrosées et les plus fraîches, naturellement peu propices à la

\section{Vers une renaissance des vins celtes?}

Disserter à propos de vins et vignobles en Bretagne au Moyen Âge avec Julien Bachelier (dans ce volume) permet de nous remémorer cette histoire de la diffusion de la vigne liée à celle du culte chrétien dans un contexte d'optimum climatique favorable. Dans cette partie atlantique plus fraîche et humide de l'Europe, la vigne constitue toujours un produit d'appel important, symbolique et patrimonial, alors qu'elle connaît une quasi-ellipse historique depuis les temps modernes. La vigne a alors disparu sous les coups de boutoir conjoints du Petit âge glaciaire, de la spécialisation des régions agricoles et de la Révolution industrielle. Malgré cela, il reste près de 800 ha de vigne à la mi-XIX siècle en Bretagne, ce qui témoigne de l'ancienne vocation viticole de ce territoire. Il faut attendre l'épidémie de phylloxéra pour voir presque disparaitre alors le vignoble breton. Comme dans toutes les régions où la vigne s'est éteinte à un moment, c'est souvent par la toponymie que son souvenir subsiste, par exemple dans la vallée de la Rance. Et même si la région administrative d'aujourd'hui communique sur le vignoble breton à travers la vaste appellation Muscadet autour de Nantes et de l'estuaire de la Loire, il reste que la Bretagne n’est plus du tout associée à l'idée de viticulture pour le grand public.

C'est dans ce contexte alliant représentations, patrimonialisation autour de la vigne, et mise en avant des conséquences du réchauffement climatique, que l'on observe une renaissance de la culture de la vigne en Bretagne depuis quelques années, auquel s'ajoute une réflexion sur ce qu'a été ce vignoble celte par le passé, peut-être pour l'inscrire dans un héritage plus global. La culture de la vigne semble remonter vers le nord, afin de (re)coloniser des terroirs au climat potentiellement plus favorable dans un futur proche, en Ille-et-Vilaine par exemple. Beaucoup de ces initiatives sont associatives, communales, et profitent du fait que depuis 2016, l'ONIVins, (Office National Interprofessionnel des Vins), gérant la production de «Vins de pays », permet à nouveau de créer de nouveaux vignobles. Depuis les années 2000, entre 100 et 200 viticulteurs sont déclarés dans les départements bretons où ils cultivent encore des variétés hybrides témoins de l'immédiat après phylloxéra, et aussi des cépages classiques comme le Pinot noir, le Chardonnay ou le Chenin, cépage de la partie estuarienne bretonne de la vallée de la Loire. C'est aujourd'hui encore une viticulture de témoignage, visant à faire revivre du patrimoine, à vocation culturelle et oenotouristique, à l'image des vignobles relictuels d'Île de France. 
viticulture, l'évolution vers une économie d'échange a eu raison de cette dernière, à part peut-être dans la Galice espagnole, dont la partie sud au contact du Portugal a gardé quelques traditions de production. De la même façon qu'en Bretagne hexagonale, la Grande-Bretagne d'outre-manche a perdu beaucoup de sa tradition vitivinicole par rapport aux siècles passés. La vigne y était répandue pratiquement sur tout le territoire de l'Angleterre et du Pays de Galles, avec un tropisme climatique vers le sud du pays, comme en témoigne encore la répartition actuelle, éclatée et relictuelle (figure 1).

Malgré des difficultés de maturation, de gels tardifs, d'irrégularités des rendements, la viticulture concerne un peu plus de 100 wineries et près de 500 viticulteurs. Certains ne possèdent qu'une treille et ne produisent que symboliquement. La production est centrée sur des vins blancs $(90 \%)$ et des vins effervescents plus faciles à élaborer dans cette ambiance fraîche et humide. L'investissement dans

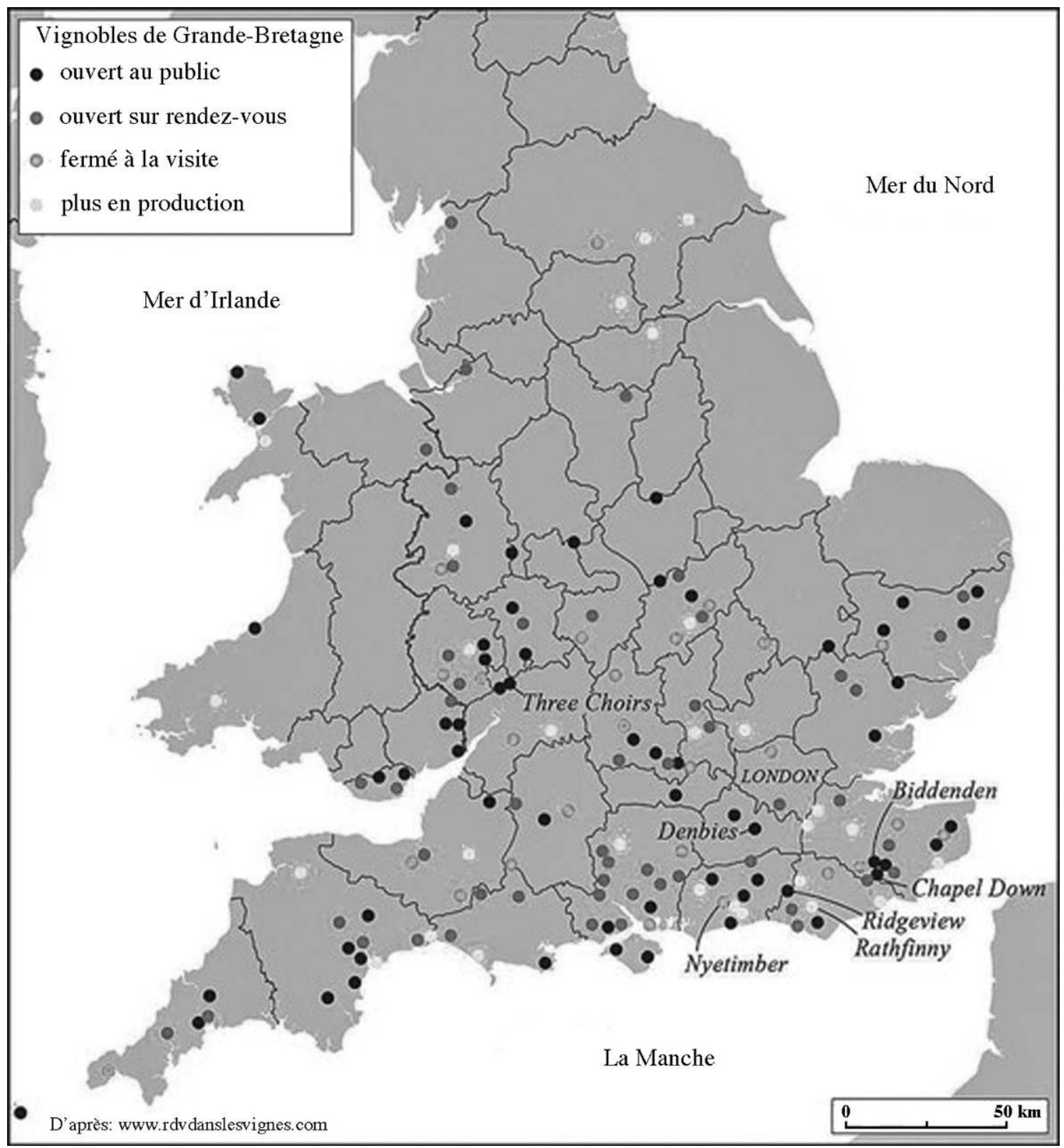

Figure 1 : Vignobles de Grande-Bretagne Vineyards of Great-Britain (Clout, 2013) 
les promesses du réchauffement climatique a poussé le vignoble à doubler depuis 15 ans, de grandes maisons champenoises y ayant investi par exemple. D'autant plus que les Britanniques accordent des valeurs symboliques importantes aux vins, se rangeant dans l'esprit anglo-saxon, qui nous gagne aussi, qui l'associe à un certain style de vie de classe supérieure, réservé à des amateurs éclairés, faisant référence à un univers complexe, subtil, raffiné, d'alliances de goûts et de plats cuisinés par exemple, au contraire de la consommation de la bière ou d'alcools forts, associés à des cultures plus populaires.

Ces vignobles du nord-ouest demeurent donc particuliers, entre production symbolique, patrimonialisation, représentation et renaissance.

\section{Diffusion et localisation des vignobles atlantiques}

Introduite au-delà des colonnes d'Hercule dès les temps antiques par les romains conquérants de l'Afrique du nord, la vigne a sans doute colonisé très tôt à l'ère chrétienne les rivages atlantiques de l'Europe et de l'Afrique du nord, remontant en latitude dans les îles britanniques, jusqu'au mur d'Adrien au Royaume-Uni, jusqu'au Danemark dans un contexte de diffusion du culte chrétien et d'un optimum climatique médiéval favorable. Avec les grandes découvertes de l'époque moderne, les rivages américains des deux hémisphères, additionnés par l'Afrique australe au XVII ${ }^{\mathrm{e}}$ siècle, vont contribuer à la conquête de la variété européenne vitis vinifera. L'ouverture des routes commerciales, l'influence des grands ports, les besoins en approvisionnement en vin ont favorisé la mise en place de ces vignobles atlantiques littoraux et rétro littoraux. Ils ont acquis au fil des siècles des notoriétés, des singularités, qu'ils soient abrités par les littoraux continentaux ou des milieux insulaires.

Côté américain, la vigne déroule sa présence de l'embouchure du Saint-Laurent au Canada à celle de l'Uruguay-Paraná entre l'Uruguay et l'Argentine, en passant par la côte nord-est des États-Unis et le sud du Brésil. Côté européen et africain, de l'Angleterre au Cap occidental en Afrique du Sud, en passant par les littoraux de l'Europe continentale, le Maroc et les nombreuses îles qui les bordent, la vigne jouit d'une présence reconnue, parfois à fort poids socio-économique, complétée de valeurs patrimoniales et paysagères. Ceci fonde des poten- tiels oenotouristiques remarquables comme par exemple l'île de Lanzarote dans les Canaries, avec le contraste de ses ceps au feuillage vert abrités dans des vasques cerclés de murets en pierre, creusées dans les sables noirs volcaniques.

Il peut donc paraître hasardeux de définir une typologie de ces vignobles atlantiques, tant leur disparité est apparente. Mais se distinguent les plus anciens, européens, insulaires et issus de la colonisation de l'Afrique du nord et de ses rivages. Peut être également mise en avant la particularité des vignobles nord-américains, plus récents, datant de l'époque moderne mais surtout actifs économiquement depuis la seconde moitié du $\mathrm{xx}^{\mathrm{e}}$ siècle, surfant sur l'effet de niche et l'attrait des conditions extrêmes comme au Canada, ou évoquant toujours un mélange des genres entre tradition et modernité aux États-Unis, le Nord-est viticole se situant en dehors des grandes régions viticoles de ce pays comme la Californie et la vallée de Napa.

Les vignobles atlantiques sud-américains apparaissent plus traditionnels, dans la lignée des européens, culture latine vitivinicole oblige peutêtre. Quant aux vignes de l'Afrique australe, elles constituent une catégorie à part, éloignée par les eaux, éloignée aussi par des éléments socioculturels importants. Mais il demeure intéressant de les appréhender par grandes zones bioclimatiques par exemple, ou en fonction de leur histoire, appartenant à l'Ancien ou au Nouveau monde vitivinicole.

En Europe, depuis la refondation de la filière viticole après l'épidémie de phylloxéra fin XIX ${ }^{\mathrm{e}}$, début $\mathrm{XX}^{\mathrm{e}}$ siècle, il est admis que la viticulture de qualité, considérée comme digne de donner des raisins suffisamment sucrés pour une bonne fermentation alcoolique, ne s'aventure plus guère au nord de l'isotherme des températures moyennes minimales de juillet de $10{ }^{\circ} \mathrm{C}$. Cette ligne passe actuellement juste au nord de la vallée aval de la Loire, au sud de Paris et effleure le nord de la Champagne. Les vignobles plus au nord sont tombés en désuétude, comme au Royaume-Uni, ou ont su renaître avec des produits de niche spécifiques alliés à une solide communication comme les vins de glace canadiens à partir des années 1980, (Vélasco-Graciet et al., 2006).

Les vignobles atlantiques européens sont, comme dans de nombreux cas, commercialement liés à un fleuve et une embouchure maritime assurant historiquement le transport du vin. 
Le reste de la façade atlantique européenne fait partie de l'épicentre des pays viticoles contemporains, avec la basse vallée de la Loire, les littoraux charentais avec le Cognac, le Bordelais, vignobles à réputations mondiales. Les vignobles béarnais et basques ne sont pas négligeables, Jurançon, Irouleguy ou Txakoli si la frontière espagnole est franchie. La vigne dans l'Espagne atlantique est moins présente que dans le reste de la péninsule, mais comprend la fameuse Rioja. Quant au Portugal, l'emprise viticole est en totalité sous influence atlantique, avec la réputation du Vinho Verde et surtout du Porto associé à la vallée du Douro et sa ville portuaire éponyme (figure 2).

Si on franchit la Méditerranée, au-delà du détroit de Gibraltar, la viticulture atlantique est encore présente au Maroc, y compris en tant que production de raisin de cuve destinée à la fermentation alcoolique, malgré le tabou culturel sur la consommation d'alcool. Tout l'ouest du pays est ponctuellement concerné, de la province de Kenitra au nord-ouest avec les vignobles de Gharb-Chardja-Beni Hassen à celle de Saft au sud-ouest avec les vignobles de Doukhala-Abda, en passant par Fez-Boulmane et Meknès-Tafilalet aux contreforts de l'Atlas.

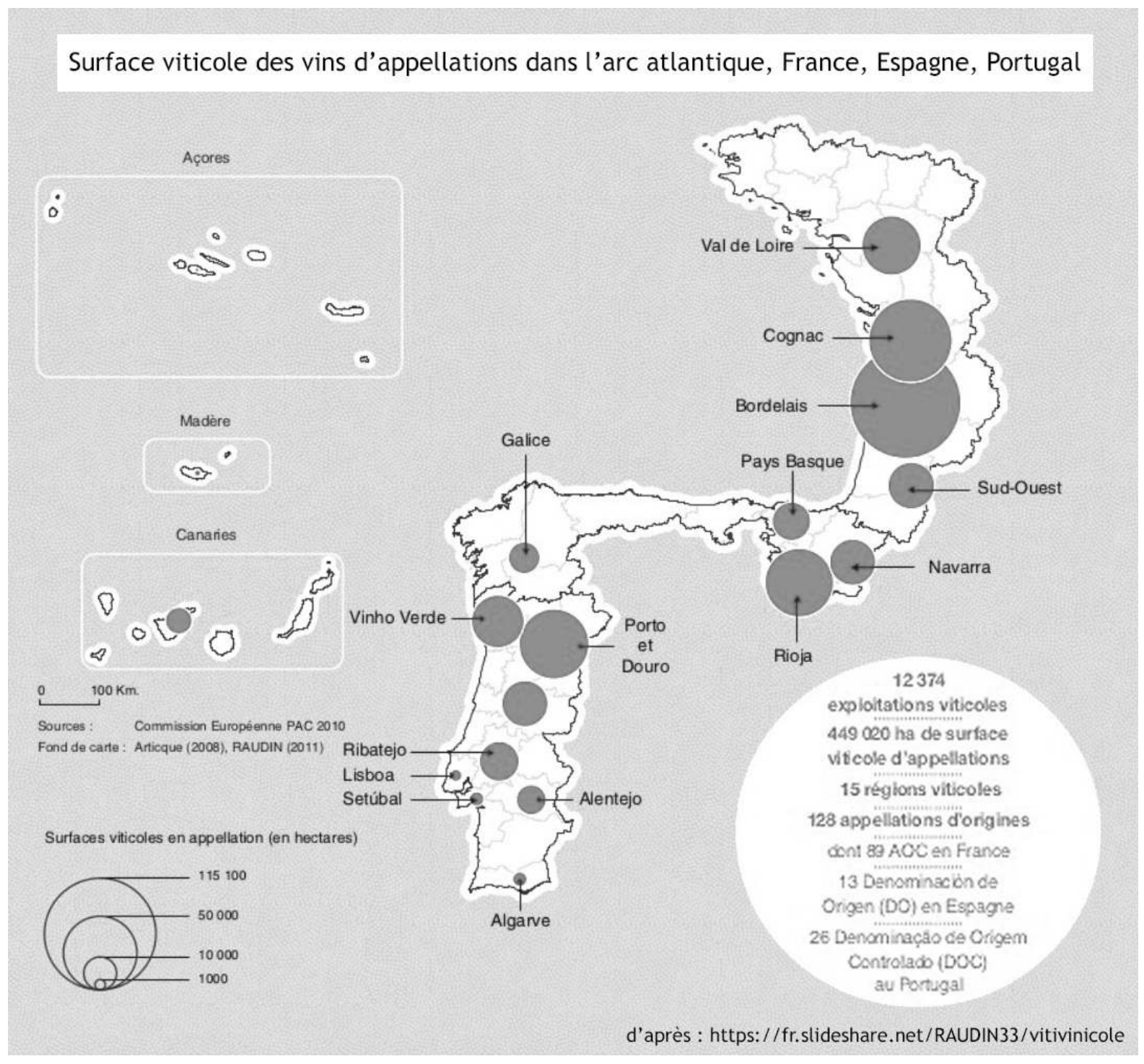

Figure 2 : Surfaces viticoles des vins d'appellations dans l'arc atlantique : France, Espagne, Portugal, (Pacquelin et al., 2012) Wine-growing areas for guaranteed vintages wines in the Atlantic Arc: France, Spain, Portugal 


\section{L'image du vin portugais à travers le Porto}

Il suffit d'évoquer le remariage d'Aliénor d'Aquitaine en 1152, avec Henri II Plantagenêt roi d'Angleterre, pour comprendre l'essor de Bordeaux, vignoble et port anglo-français viticole pendant 850 ans. Cette alliance entre grands vignobles et grands cours d'eau exutoires marchands, chère à Roger Dion, s'observe partout, perdure et marque aussi, par exemple, toute la région du Porto, sur la majeure partie du fleuve Douro, avec son port éponyme.

Dans le cas présent, on retrouve le problème structurant la viticulture et le vin en tant que produit toujours fortement échangé, face à son statut de denrée périssable lors de transports aux longs cours. Ceci commande encore la vision de la qualité d'un vin, malgré la révolution des transports au XIX ${ }^{\mathrm{e}}$. Le vin qualitatif est avant tout celui qui se conserve.

Rares sont alors les breuvages qui atteignent la récolte suivante de raisins sans s'oxyder et devenir du vinaigre. Il faudra attendre les travaux de Chaptal et de Pasteur pour mieux maîtriser les fermentations. Pendant des siècles, les sociétés vont rivaliser d'imagination pour pallier la carence de sucre pouvant se transformer de manière ménagée en alcool et assurer la continuité du vin, ou simplement rajouter des produits pour masquer les goûts vinaigrés.

Au XIII ${ }^{\mathrm{e}}$ siècle, la tradition attribue, à tort ou à raison, à Arnaud de Villanova, un médecin valencien, la maîtrise du mutage par rajout d'alcool dans le moût du raisin. Cette pratique assurera la pérennisation des vins de bien des régions, à l'embouchure de la Charente par le Pineau éponyme, le long du Golfe du Lion avec les Muscats, Maury ou autres Banyuls, dans la vallée du Douro avec la création du Porto, qui essaime jusque dans la région semi-aride du Klein Karoo en Afrique du Sud, où sont produits des vins mutés sous le nom de « Port ». Le défi de la qualité du produit passant par sa capacité à se conserver lors de stockages, de trajets, d'expositions prolongées au soleil pouvant dépasser une année avant d'être consommé est relevé. Et il n'est donc pas étonnant de constater le développement économique pluriséculaire, la consommation internationale, l'apparition de tradition, de patrimoine, de paysages remarquables autour d'un produit comme le Porto.

Tout entier tourné vers l'Atlantique, le Portugal détient une tradition viticole parmi les plus vivaces au monde, $11^{\mathrm{e}}$ producteur mondial, avec un marché intérieur qui compte, les Portugais sont les premiers buveurs de vin mondiaux avec près de 60 litres par personne et par an. Il faut néanmoins exporter la majeure partie de la production vu le faible volume de population du pays. Voilà pourquoi le marché français, comme l'évoque l'article de Philippe Baumert dans ce numéro, est prometteur d'un potentiel de consommateurs tout sauf négligeable, ( $2^{\mathrm{e}}$ mondiaux avec près de 50 litres de vin par personne et par an) pour le Porto.

Paradoxalement peu connu en France, où de plus la consommation des vins dits d'apéritifs ou de desserts ${ }^{1}$ est en perte de vitesse depuis plusieurs décennies, notre pays est le premier marché d'exportation du vin de Porto, près de 190000 hl, loin devant les marchés néerlandais (110000 hl) et britannique (90000 hl). Cette configuration date du début du siècle dernier avec l'engouement de ce genre de boissons apéritives, au point d'avoir détrôné le Royaume-Uni, premier client multiséculaire ayant structuré la route maritime commerciale et le produit lui-même. Ce marché français est d'autant plus original car méconnu, à l'image un peu désuète et offrant peu de place aux Portos les plus qualitatifs. Mais les atouts avancés, une mise en avant d'un patrimoine paysager sans égal et la construction de véritables vins de terroirs, amènent le Porto à jouer dans une nouvelle cour mondialisée des échanges vitivinicoles.

Ici finit l'Ancien monde viticole héritier des colonisations romaines, chrétiennes et musulmanes; lui répond depuis 30 ou 40 ans maintenant un Nouveau monde, américain et d'Afrique australe. La vigne

1. Les termes « muté » ou « vin doux naturel » étant peu connus du grand public. uvifère y a été introduite au rythme des colonisations européennes mais c'est l'ouverture libérale, des changements de consommation, qui ont porté ces nouveaux vignobles sur le devant de la scène. Il se trouve une dissymétrie évidente entre les deux rives de l'Atlantique. Le vignoble canadien occupe les 
rives du Saint-Laurent, en face de son homologue du nord-est des États-Unis, où la vigne se développe en dehors des régions célèbres de la Californie et de l'Oregon à l'ouest du pays. Côté atlantique, les états de New York, du Massachusetts, du Connecticut, du New Jersey, de Pennsylvanie, de l'Ohio, de Virginie et même de Caroline du Nord abritent quantité de vignobles, mais les traditions vitivinicoles et les superficies sont sans commune mesure par rapport aux rivages européens. Ici les habitudes de consommation sont passées au $\mathrm{Xx}^{\mathrm{e}}$ siècle de la prohibition à la quasi magnification du vin, avec la médiatisation il y a près de 40 ans du "French paradox », (Perdue, 1995), où est mis en évidence le rôle antioxydant des composés colorants du vin, consommé dans des proportions bien maîtrisées.

Hors des régions atlantiques tempérées, la vigne se développe dans des pays intertropicaux, même si elle y est plutôt cultivée en altitude pour pallier les effets climatiques. Des vignobles de cuve non négligeables existent au Mexique, au Venezuela, à Cuba, en Colombie, dans les parties tropicales du Brésil, mais aussi dans l'île de Cap Vert, (tableau 1), (Salomon, 2005).

Enfin, ces vignobles atlantiques se déclinent sous forme insulaire : Ré, Oléron, Canaries, Açores, Madère, Cap vert, où l'effet de niche est poussé à son paroxysme, avec l'image de l'île, ses paysages, sa réputation, son œnotourisme. C'est là, par exemple, que le postmodernisme et son corollaire du relativisme apparaissent dans le monde vitivinicole d'aujourd'hui, avec une approche du vin qui n'est plus uniquement un produit à découvrir par une simple dégustation. Quand un visiteur arrive dans un chai, il ne vient plus que pour goûter et acheter, et éventuellement visiter. Il est attiré parfois par l'architecture iconoclaste des bâtiments valorisée comme patrimoine bâti mais également viticole (ColignonDarmaillac, 2009; Schirmer, 2013; Rouvellac, 2014); il se voit proposer, une diversité de services allant de la restauration, à l'hébergement, aux expositions et manifestations artistiques dans le chai ou dans les vignes, aux sports de pleine nature... quand ce n'est pas de participer symboliquement aux travaux de la vigne. C'est une approche intégrée qui est souvent privilégiée aujourd'hui, où le vin est relativisé et accompagne des activités culturelles et touristiques. À l'Île de Ré, la production de vins, comme celle de pommes de terre, toutes deux label- lisées AOP, surfent sur l'image touristique « haut-degamme » de l'île, sans que la qualité intrinsèque du produit soit remise en question. À Lanzarote, dans l'archipel espagnol des Canaries, le paysage volcanoviticole déjà cité joue à lui seul pour la réputation oenotouristique de l'île, qu'importe le résultat dans le verre du consommateur.

Produit marchand à forte valeur ajoutée commerciale et symbolique, le vin est devenu depuis les années 1980 un produit comptant dans la politique d'exportation des pays viticoles du Nouveau monde, construit au départ en opposition à l'Ancien monde européen. Mais depuis de nombreuses années, les vins de cépages, mis en avant par le Nouveau monde et jugés plus facilement reproductibles en opposition aux vins de terroirs de l'Ancien monde, plus en phase avec les systèmes d'appellation d'origines, avec leur territoire, leur tradition d'assemblage parfois, se nourrissent mutuellement de chaque côté de l'Océan atlantique. Et les pays du Nouveau monde vitivinicole interfèrent à leur manière dans la nouvelle donne reliant viticulture et réchauffement climatique.

\section{LE RÉCHAUFFEMENT CLIMATIQUE : UN FACTEUR DE CHANGEMENT QUI REBAT LES CARTES?}

\section{Une production et une consommation internationale et concurrentielle}

Le contexte atlantique renvoie à cette opposition classique entre Ancien et Nouveau monde viticole. Les États-Unis constituent le $4^{\mathrm{e}}$ producteur mondial, (24 Mhl de vin en 2018) et sont les premiers consommateurs en volume depuis 2013, (38 Mhl en 2018), l'Argentine est le $5^{\mathrm{e}}$ producteur mondial, l'Afrique du Sud le 9e , le Brésil le 15e.

Si la zone tempérée sud est atteinte, ce sont des pays aux productions viticoles non négligeables, comme le Brésil ou l'Uruguay, ou très importants en volumes et en superficies sur l'échiquier vitivinicole mondial, comme l'Argentine ou l'Afrique du Sud, qui sont à considérer. Ces pays ont pour caractéristique première d'avoir peu de marché intérieur pour des raisons culturelles, économiques, ou même ethniques comme en Afrique du Sud où le vin est fortement associé à des modes de consommation de «blancs » aisés dans une société postapartheid qui évolue lentement (Rouvellac et al., 2013). En Amérique du 


\begin{tabular}{|l|l|l|l|}
\hline Pays & Principales régions concernées & $\begin{array}{l}\text { Superficie en } \\
\text { vigne ha }\end{array}$ & $\begin{array}{l}\text { Production vin } \\
\mathbf{1 0 0 0} \text { hl }\end{array}$ \\
\hline Canada & Saint-Laurent & 12627 & 548 \\
\hline États-Unis & Côte nord-est & 439000 & 23715 \\
\hline Mexique & & 31420 & 404 \\
\hline Cuba & & 1816 & 117 \\
\hline Venezuela & & 1247 & $?$ \\
\hline Brésil & Rio Grande do Sul & 86408 & 1257 \\
\hline Colombie & & 2592 & $?$ \\
\hline Argentine & Mendoza, San Juan & 224258 & 9447 \\
\hline Uruguay & Uruguay-Paraná & 7251 & 755 \\
\hline Royaume-Uni & Angleterre, Pays de Galles & 2000 & 45 \\
\hline France & $\begin{array}{l}\text { Vallée aval de la Loire, de la Charente, de la Garonne, } \\
\text { Ré, Oléron, Landes, Pays basque }\end{array}$ & 786054 & 45367 \\
\hline Espagne & Péninsule atlantique, Canaries & & \\
\hline Portugal & Partie atlantique, Madères, Açores, & 975270 & 39670 \\
\hline Cap Vert & Fogo & 195284 & 6010 \\
\hline Maroc & Côte Nord-ouest & 120 & $?$ \\
\hline Namibie & & 49455 & 349 \\
\hline Afrique du Sud & Cap occidental & 7320 & $?$ \\
\hline
\end{tabular}

Tableau 1 : Pays viticoles bordiers de l'océan atlantique, (OIV, 2016). En italique : données non exclusivement atlantiques Wine-growing countries bordering the Atlantic Ocean

Sud, peu de personnes ont accès économiquement à cette boisson qui plus est, ne constitue pas un produit culturel, notamment pour les Amérindiens. Pour tous ces pays-là, la devise ou le ressort de la filière vitivinicole est donc " exporter ou disparaître », ce qui explique leur agressivité à l'exportation et leur compétitivité grâce à des coûts de mains d'œuvre très bon marché par rapport à l'Europe.

Situé aux pieds des Andes dans une ambiance semi-désertique, le vignoble argentin est surtout atlantique par ses débouchés commerciaux. La particularité sur laquelle il communique tient à un encépagement original moins classique, constitué à $20 \%$ (soit près de 45000 ha) de Malbec, ou Cot noir, variété française cultivée presque uniquement dans le sud-ouest de la France, dans la moyenne vallée du Lot et dans le vignoble AOP Cahors (4200 ha environ, 5000 ha pour toute la France). Le vignoble uruguayen communique aussi particulièrement sur le cépage Tannat, qui couvre 3000 ha en France, dans le Sud-Ouest également où il fournit l'ossature de l'AOP Madiran. Mais il assure $60 \%$ de la production et près de 2000 ha en Uruguay, où il est désigné par le nom de son introducteur basque «Harriague » vers 1870. Dans les deux cas, l'immigration post-phylloxérique de viticulteurs de ces régions françaises explique en partie la diffusion de ces cépages qui aujourd'hui sont plus étendus que dans leur berceau originel, et même emblématique de leur vignoble du Nouveau monde. Cet encépagement désormais plus étendu à l'ouest de l'atlantique qu'à l'est marque cet effet boomerang où le Nouveau monde, semble faire mieux, et plus grand, que l'ancien.

Les pays viticoles d'Amérique du Sud communiquent généralement aussi sur l'absence de phylloxéra dans leur vigne, prétextant que leur vignoble ainsi non greffé est plus sain, plus naturel, exempt de maladies contrairement aux vignobles européens. L'examen rapide des vignes apprend très facilement qu'au moins la moitié du vignoble argentin, par exemple, est greffé, ce qui laisse douter sur l'absence du phylloxéra sur tout le territoire.

Plus atypique est le vignoble d'Afrique du Sud, débordant jusqu'à l'ancienne colonie de ce pays, la Namibie. Atypique par exemple car il peut être 
daté précisément, la vigne a été introduite en 1652 par la compagnie maritime néerlandaise des Indes orientales, voulant fonder une étape pour se ravitailler en produits frais (et en vin), à proximité du Cap de Bonne Espérance. Les Néerlandais fondent alors le vignoble de Constantia, qui existe toujours sur les hauteurs de Cap Town, donnant de fameux vins doux à base de Muscat d'Alexandrie. Atypique car ici les Français sont clairement identifiés dans l'histoire viticole, par l'endroit qui a été dévolu aux huguenots chassés par la révocation de l'Édit de Nantes en 1685, la vallée de Franschhoek (le " coin des Français » en afrikaans), non loin de Stellenbosch, épicentre du vignoble. Atypique enfin par le contexte de l'apartheid et de la transition postapartheid, où pendant des décennies au $\mathrm{Xx}^{\mathrm{e}}$ siècle des populations noires ou métisses ont constitué une main-d'œuvre, dans des conditions proches parfois de la servitude, dans les vignes dont la production était sous embargo international, et continuent encore de travailler aujourd'hui dans des conditions très précaires. Dans aucun pays viticole le vin n'est autant assimilé à un marqueur culturel et ethnique, associé à la société «blanche ». L’Afrique du Sud est depuis des siècles un grand pays viticole, pionnier dans le mouvement coopératif avec une des premières caves au monde fondée à Tulbach en 1905 , ayant même créé dans les années 1920 un cépage emblématique par le croisement du Pinot noir et du Cinsault, le Pinotage. Mais son isolement à la fois politique lié à l'apartheid, géographique et culturel l'a aussi condamné à exporter l'essentiel de sa production (Rouvellac et al., op. cit.).

\section{Le réchauffement climatique relativise l'opposition Ancien / Nouveau monde viticole}

L'Europe adopte à son tour un tournant prohibitionniste, (bien que l'Espagne ait décrété en 2003 le vin comme un aliment pour contourner les lois européennes sur la publicité pour les produits alcoolisés), et continue d'associer vin et alcoolisme, alors que la consommation de celui-ci en est chute libre depuis un siècle, (150 l/personne/an en 1900, 50 à la fin des années 2010 en France). L'opposition Ancien/Nouveau monde vitivinicole évolue vers une complémentarité depuis plusieurs années. Loin de l'association vin aliment ou vin alcoolisme, le Nouveau monde nous renvoie de nouvelles associations entre vins et plaisirs de consommer, vins et tourisme, vins et culture, autant de nouveaux médias pour communiquer à propos du vin dans un contexte répressif. Ce boomerang culturel s'accompagne certes d'une uniformisation et d'une standardisation des goûts avec la mode des vins de cépages et des vins de marque amenée par ces nouveaux pays producteurs. Mais depuis plusieurs décennies, les pays du Nouveau monde élaborent des politiques de vins de terroirs et d'appellations, et pas uniquement pour pénétrer les marchés européens. L'Europe a heureusement aussi adopté les vins de marque et de terroirs et essaie de jouer entre les produits d'entrée de gamme et ceux plus élitistes, en lien avec l'évolution de demande du consommateur qui boit nettement moins de vin, mais boit mieux, veut connaître l'origine du produit, son histoire, son contexte culturel.

Le vin devient une marque de différenciation sociale, sa consommation a changé et ses lieux de consommation aussi. Il se boit un peu moins à la maison, plus lors de sorties, lors d'occasions plus festives. Le rapport à l'alcool dans cette boisson aussi a changé. Les apéritifs vineux, les vins titrant plus de 14 ou 15 degrés d'alcool font moins recette, le consommateur et la consommatrice préfèrent des vins plus légers, d'où le succès des blancs, des rosés, des «mixed » vins et fruits plus sucrés, moins concentrés, mais aussi plus accessibles pour une clientèle moins avertie qui craint une forme de snobisme que certains associent au vin.

Même si les conditions culturelles, sociales, économiques sont importantes, parfois primordiales, pour produire des raisins de qualité et potentiellement un vin qui va de même, les conditions naturelles ne le sont pas moins. Nous avons vu que le vin constituait un produit d'appel fascinant facilement l'imaginaire dans la conception des lanceurs d'alerte sur les dangers du changement climatique. Nous avons vu également que ces prévisions créent un effet d'opportunisme pour des acteurs dans des régions qui ne sont pas, ou peu, ou presque plus viticoles actuellement. Beaucoup de pays des hautes latitudes tempérées espèrent tirer profit finalement du réchauffement au plan viticole, comme le Royaume-Uni au premier plan, mais aussi le Canada, ou même la Norvège, l'Islande... Ces pays paraissent jalouser les régions productrices 
en voulant s'appropriant une partie de l'image patrimoniale et de style de vie agréable donnée par les pays viticoles. La dialectique Ancien et Nouveau monde (est/ouest Atlantique) par rapport aux vins et vignobles pourrait-elle à terme évoluer vers une dialectique sud / nord?

Ainsi le Royaume-Uni prétend qu'il pourra héberger la Bourgogne et la Champagne. Certes, mais à quelles échéances et à quel rythme? Même s'il pourrait être rapide comme nous le montre la figure 3, la question des marchés reste ouverte. Car, en même temps, les anciennes régions européennes ne verront peut-être pas leurs vignobles disparaître d'un coup, mais se transformer, s'adapter. Et dans des échanges mondialisés, d'autres régions froides, atlantiques ou non, deviendront elles aussi viticoles et concurrentielles. Il suffit de penser aux milliers d'hectares que les Chinois sont en train de planter dans les déserts de Mongolie intérieure, dans le Ningxia par exemple.

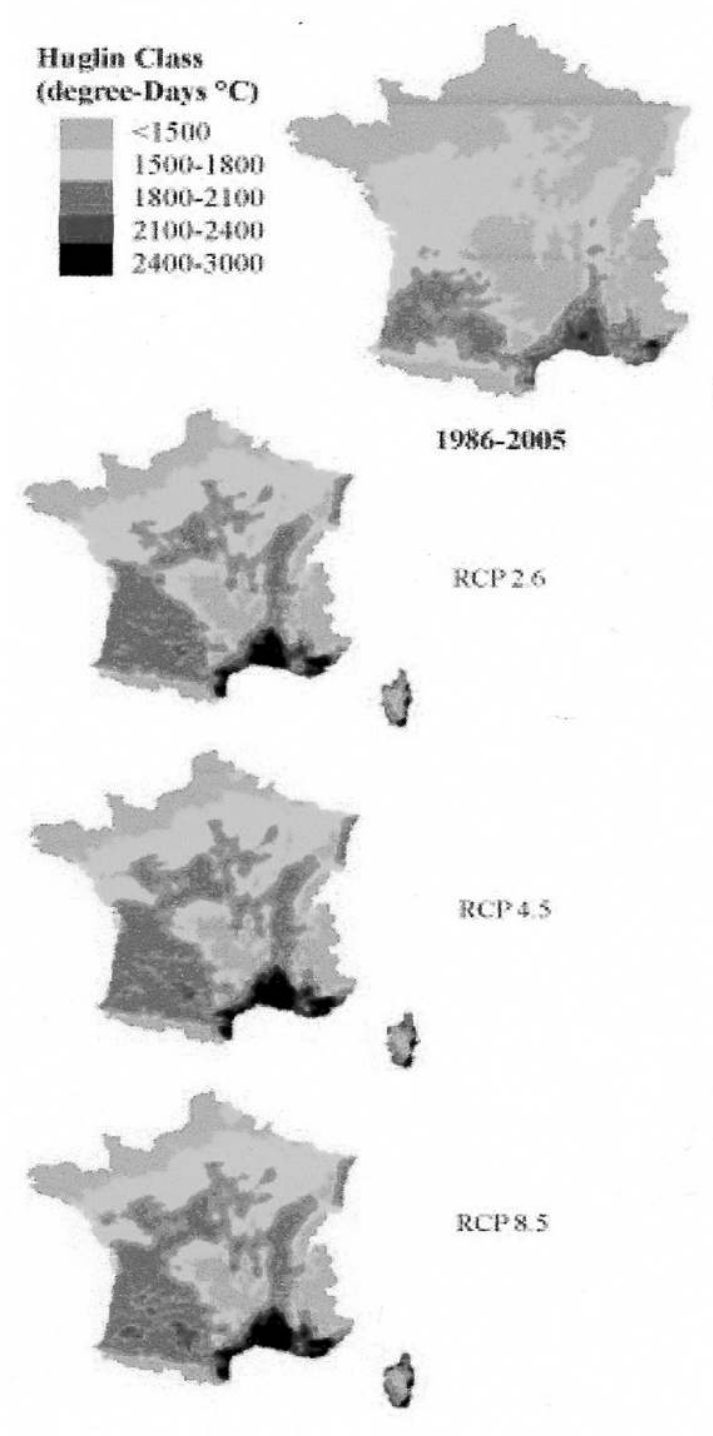

2031-2050

\begin{tabular}{|c|c|c|}
\hline Class & ${ }^{8} \mathrm{C}$ & Ex:armples \\
\hline $\begin{array}{l}\text { Very } \\
\text { warm }\end{array}$ & III -3200 & $\begin{array}{c}\text { Sho Frateises Valley } \\
\text { (Brusal) }\end{array}$ \\
\hline warm & $2400 \leq \mathrm{IH} \leq 3001$ & $\begin{array}{l}\text { Malaga (Spain) } \\
\text { Marsala (thaly) }\end{array}$ \\
\hline $\begin{array}{c}\text { Warm } \\
\text { teasperate }\end{array}$ & $2100=\mathrm{IH}=2 \mathrm{tan}$ & $\begin{array}{c}\text { Napa (USA) } \\
\text { Montpellier (France) }\end{array}$ \\
\hline semperate & $1800=1 \mathrm{HH} 2100$ & Paul, Bosdesux (France) \\
\hline cold & $1500 \leq 111 \leq 1800$ & Colnas, Angurs (france) \\
\hline Very enld & $\mathrm{HH} \leq 1580$ & $\begin{array}{l}\text { Quebse (Cansda) } \\
\text { London (Eapland) }\end{array}$ \\
\hline
\end{tabular}

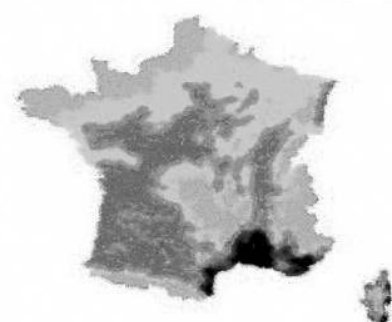

$\mathrm{RCP} 26$

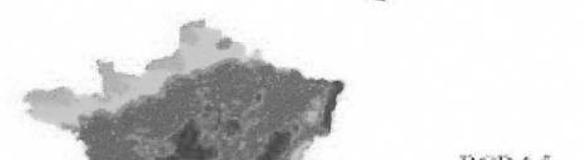

$\mathrm{RCP} 4.5$

RCP8.5

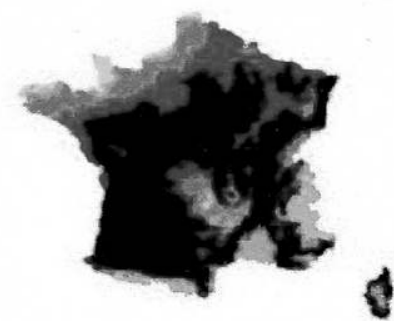

$2081-2100$

Mean Huglin Index classes through RCP scenarios. (sources: DRIAS) (Quénol et alii, 2017)

Figure 3 : Évolution de la viticulture en France au XXI ${ }^{\mathrm{e}}$ siècle. (Quénol et al., 2017)

Evolution of viticulture in France in the 21st century 
Au sud de la province du Cap occidental en Afrique du Sud, vers le Cap des Aiguilles, pointe plus australe que le Cap de Bonne espérance, les viticulteurs essayent de profiter au maximum de la fraîcheur océanique en plantant de la vigne, arrachant les pommeraies initiales. Est-ce seulement pour rechercher des températures moins chaudes ou élaborer des cuvées moins alcooleuses, voulues actuellement par l'évolution des goûts des consommateurs? Difficile de trancher, sûrement un peu pour ces deux raisons. Voilà pour l'instant un exemple concret et localisé, en phase avec un marché, de corrélation entre réchauffement climatique et évolution de la vitiviniculture. Beaucoup de vignobles dans le monde en sont encore à l'heure du diagnostic et de la réflexion; d'autres commencent à passer à l'action, grâce aux études lancées par les chercheurs en climatologie, agronomie, géographie environnementale et/ou sociale.

Ce premier numéro thématique présente ainsi trois exemples de stratégies d'adaptations à travers les vignobles du Val de Loire en France et de Mendoza en Argentine. Les deux premiers mettent l'accent sur des enquêtes auprès des acteurs de la filière vitivinicole, le troisième le fait aussi mais à travers une approche de modélisation géomatique.

À partir d'un travail d'élaboration d'indicateurs climatiques et agro-climatiques pertinents pour aider les viticulteurs à anticiper les effets des évolutions climatiques sur leurs pratiques, François Bertrand, Corentin Thermes, et Isabelle La Jeunesse développent des actions d'adaptation au changement climatique à l'échelle de l'exploitation, pour réagir au changement actuel ou anticiper celui-ci. Ensuite, des trajectoires d'adaptation à moyen et long termes sont co-construites avec trois exploitations-pilotes, avec pour objectif le maintien de la viabilité économique des exploitations. Dans la même perceptive, les mêmes auteurs proposent d'explorer différentes formes de déplacements et de réorganisations provoqués par le changement climatique. En s'appuyant sur l'étude de l'AOC Ventoux et de plusieurs AOC du centre Val de Loire, l'article analyse comment ces options peuvent nourrir des stratégies d'adaptation au changement climatique en s'appuyant sur les résultats du projet LACCAVE, (Impacts et adaptations à long terme de la filière vitivinicole au changement climatique), porté par l'Institut National de la Recherche Agronomique, qui veut préciser les impacts, explorer et tester des adaptations possibles par des vignobles face au changement climatique. Ce projet souligne quatre points impactant la viticulture dans le futur : l'avancée des stades phénologiques de la vigne qui se poursuit, le changement de l'évolution des caractéristiques des vins, la modification des bilans hydriques des vignobles, avec des effets contrastés selon les régions - un effet négatif sur les rendements au sud et une évolution de la pression des maladies au nord -, et des effets importants sur la répartition géographique possible des vignobles. L'article s'appuie sur le scénario « Nomade », un de ceux définis par le programme LACCAVE, insistant sur le contexte qui pousse la vigne à être délocalisée dans des terroirs qui seront appelés à remplacer de grandes appellations ou à migrer en plaine pour trouver davantage d'eau.

Cette dernière question de la ressource en eau est un des thèmes important du troisième article de Cyril Tissot, Hervé Quenol, Mathias Rouan sur le changement climatique dans le vignoble argentin à Mendoza. Ils présentent une approche de modélisation multi-agents visant à simuler les relations entre climat local, cycle phénologique de la vigne et stratégies d'acteurs de productions viticoles. Cette démarche se place dans la perspective de mieux comprendre les ajustements de l'agronomie aux variations du climat. Cette approche par les systèmes multi-agent (SMA) est intéressante pour lancer des modélisations prédictives à l'échelle d'un territoire, à l'aide de programmations géomatiques composées d'agents, c'est-à-dire de critères ou d'acteurs, tous au moins partiellement autonomes, qui interagissent entre eux dans un environnement donné, selon des relations prédéfinies.

En guise de conclusion, ce premier numéro thématique essaye de balayer quelques-uns des enjeux de la filière vitivinicole mondialisée d'aujourd'hui, de l'histoire des vignobles à l'enjeu majeur du réchauffement climatique, en passant par la vitrine des vignobles que sont toujours leurs débouchés portuaires. Le changement climatique global amène des réflexions d'adaptation du local au mondial. D'imitateur, le Nouveau monde est devenu prescripteur, de production, mais aussi d'usages et de patrimonialisations, gommant un peu plus l'opposition des deux côtés de l'Atlantique. Les questions autour de l'adaptation ou de l'éventuelle disparition de vignobles sont posées. De nouveaux fronts endogènes aux vignobles atlan- 
tiques (nord/sud) vont-ils se déclarer et/ou certains vignobles anciens réapparaître?

Ceci inaugure peut-être de nouvelles mobilisations, associatives ou individuelles, pour promouvoir la vigne et son image forte dans des territoires périphériques comme les îles au large des côtes atlantiques. Et ainsi permettre de mieux définir une ou des identités à la viticulture atlantique.

\section{Bibliographie}

Clout H., 2013, An Overview of the Fluctuating Fortunes of Viticulture in England and Wales, EchoGéo, [En ligne], 23 | 2013 [https://doi.org/10.4000/echogeo.13333].

Jones G.-V., White M.-A., Cooper O.-R., Storchmann K., 2005, Climate change and global wine quality. Climatic change, 73(3), 319-343.

Lereboullet A.-L., 2014, Vulnérabilité et capacité d'adaptation au changement climatique de deux systèmes vitivinicoles méditerranéens : un cas d'étude comparatif France (Roussillon) - Australie (McLaren Vale), thèse de géographie, Université de Paris 7 Denis Diderot, 491 p.

Lignon-Darmaillac S., 2009, L'œnotourisme en France. Nouvelle valorisation des vignobles. Analyse et bilan, Bordeaux, Féret, $250 \mathrm{p}$.

Nesbitt A., Dorling S., Lovetr A., 2018, A suitability model for viticulture in England and Wales: opportunities for investment, sector growth and increased climate resilience, Journal of Land Use Science, 13:4, p. 414-438. DOI : 10.1080/1747423X.2018.1537312.

Paquelin D., Duféal M., Labranche J., Merillou F., 2012, Comment les domaines viticoles de l'arc atlantique français, espagnol et portugais s'affichent-ils sur le web? RAUDIN (Recherches Aquitaines sur les Usages pour le Développement des dispositifs Numériques, Université BordeauxMontaigne, 60 p. [https://fr.slideshare.net/RAUDIN33/ vitivinicole].

Perdue L., 1995, Le paradoxe français : réduire les risques cardiaques et vivre mieux grâce au vin et au style de vie méditerranéen, c'est le "French paradox », Avignon, A. Barthélemy, $236 \mathrm{p}$.

Quénol H., 2014, Changement climatique et terroirs viticoles. Lavoisier Tec\&doc, 460 p.

Quénol H., Garcia de Cortazar Atauri I., Bois B., Sturman A., Bonnardot V., Le Roux R., 2017, Which climatic modelling to assess climate change impacts on vineyards?, vol. 51, n 2, OENO One [https://oeno-one.eu/article/ view/1869].

Rouvellac E., Dellier J., 2013, Le vignoble sud-africain dans l'ère postapartheid, entre transformation et continuité凶, EchoGéo [En ligne] [URL : http://echogeo.revues. org/13343; DOI : 10.4000/echogeo.13343].

Rouvellac E., 2014, Les terroirs viticoles outils objectifs et subjectifs de la communication des vignobles, de l'identité des terroirs jusqu'à leur dissolution, Sud-Ouest Européen, $n^{\circ} 36,85-96$.

Salomon J.-N., 2005, Nouveaux vignobles et évolution des anciens face à la mondialisation, Les cahiers d'outre-mer, no 231-232, 397-428 [https://doi.org/10.4000/com.428].

Schirmer R., 2013, Une révolution dans les chais? Les viticulteurs du Bordelais, acteurs de leurs paysages, CNRS, UMR 5319, Regards, Université Bordeaux Montaigne, 1-9 [https:// hal.archives-ouvertes.fr/hal-00865196].

Velasco-Graciet H., Lasserre F., 2006, Le vignoble au Québec, géographie d'un rêve sous contrainte, Norois, $\mathrm{n}^{\circ} 4$, 67-82 [https://doi.org/10.4000/norois.1765]. 